\title{
Biatrial thrombi resembling myxoma regressed after prolonged anticoagulation in a patient with mitral stenosis: a case report
}

Hou Tee Lu ${ }^{1,3^{*}}$ (D), Rusli Nordin ${ }^{1}$, Norliza Othman ${ }^{2}$, Chun Ngok Choy ${ }^{3}$, Ji Yen Kam³ ${ }^{3}$ Benjamin Cheang-Leng Leo ${ }^{3}$, Gunasekaran Ramsamy ${ }^{3}$ and Teck Hwa Goh ${ }^{4}$

\begin{abstract}
Background: Many cases of cardiac masses have been reported in the literature, but in this case report we described a rare case of biatrial cardiac mass that represented a challenge for diagnosis and therapy. The differentiation between cardiac masses such as thrombi, vegetations, myxomas and other tumors is not always straightforward and an exact diagnosis is important because of its distinct treatment strategy. Transthoracic/ esophageal echocardiography and cardiac magnetic resonance play an important role in establishing the diagnosis of cardiac masses. However, no current noninvasive diagnostic tool has the ability to absolutely diagnose cardiac masses; obtaining a pathological specimen by surgical resection of cardiac masses is the only reliable method to diagnose cardiac masses accurately. Our case report is an exception in that the final diagnosis was affirmed by empirical anticoagulation therapy based on clinical judgment and noninvasive characterization of biatrial mass.

Case presentation: We described a 54-year-old Malay man with severe mitral stenosis and atrial fibrillation who presented with a biatrial mass. Transthoracic/esophageal echocardiography and cardiac magnetic resonance detected a large, homogeneous right atrial mass typical of a thrombus, and a left atrial mass adhering to interatrial septum that mimicked atrial myxoma. The risk factors, morphology, location, and characteristics of the biatrial cardiac mass indicated a diagnosis of thrombi. However, our patient declined surgery. As a result, the nature of his cardiac masses was not specified by histology. Of note, his left atrial mass was completely regressed by long-term warfarin, leaving a residual right atrial mass. Thus, we affirmed the most probable diagnosis of cardiac thrombi. During the course of treatment, he had an episode of non-fatal ischemic stroke most probably because of a thromboembolism.
\end{abstract}

Conclusions: Noninvasive characterization of cardiac mass is essential in clarifying the diagnosis and directing treatment strategy. Anticoagulation is a feasible treatment when the clinical assessment, risk factors, and imaging findings indicate a diagnosis of thrombi. After prolonged anticoagulation therapy, complete resolution of biatrial thrombi was achievable in our case.

Keywords: Cardiac mass, Thrombus, Myxoma, Atrial fibrillation, Mitral stenosis, Echocardiography, Cardiac magnetic resonance

\footnotetext{
* Correspondence: luhoutee@gmail.com; lu.hou.tee@monash.edu

${ }^{1}$ Clinical School Johor Bahru, Jeffrey Cheah School of Medicine and Health

Sciences, Monash University Malaysia, 8 Jalan Masjid Abu Bakar, 80100 Johor

Bahru, Johor, Malaysia

${ }^{3}$ Department of Cardiology, Sultanah Aminah Hospital, Jalan Abu Bakar,

80100 Johor Bahru, Johor, Malaysia

Full list of author information is available at the end of the article
} 


\section{Background}

The differentiation between cardiac masses such as thrombi, vegetation, myxomas, and other tumors is not always straightforward and an exact diagnosis is important because of its distinct treatment strategy [1]. Transthoracic echocardiography (TTE) and transesophageal echocardiography (TEE) play an important role in establishing a diagnosis of cardiac masses [2]. Cardiac magnetic resonance (CMR) offers potential advantages and is complementary to echocardiography in the evaluation of cardiac masses $[3,4]$. However, no current noninvasive diagnostic tool has the ability to absolutely diagnose cardiac masses; obtaining the pathological specimen by surgical resection of cardiac masses is the only reliable method to diagnose cardiac masses accurately [5-7].

\section{Case presentation}

In January 2011, a 54-year-old Malay man was referred to our hospital for evaluation of palpitation. On examination, his pulse rate was $85 /$ minute with irregular rhythm and his blood pressure was 120/80 $\mathrm{mmHg}$. He was afebrile. A loud first heart sound and a soft middiastolic rumbling murmur (grade 2/6 degrees) were auscultated at mitral area with no signs of heart failure. Electrocardiography revealed atrial fibrillation (AF). The laboratory results showed normal hemoglobin, white cells, and platelet counts. Renal test, liver function test, and other laboratory test results were unremarkable. His urine analysis was also normal.

TTE and TEE revealed severe mitral stenosis (MS). His mitral valve was thickened and moderately calcified, and his anterior mitral valve leaflet was "dooming" during diastole suggestive of rheumatic origin. His mitral valve area, which was estimated by two-dimensional planimetry and pressure half-time method, was $0.92 \mathrm{~cm}^{2}$ and $0.91 \mathrm{~cm}^{2}$, respectively. His peak and mean mitral valve gradients were 14 and $9 \mathrm{mmHg}$, respectively. His left atrium (LA) and right atrium (RA) were dilated. In his RA, we found a large, mobile, homogeneous round mass measuring $40 \times 35 \mathrm{~mm}$. A large LA mass with similar echogenicity to his RA mass was found adhering to interatrial septum and protruding into the left ventricle with cardiac motion (Fig. 1). We could not identify any stalk attached to the LA mass by TTE and TEE examinations. In addition, there were no identifiable masses in the left atrial appendage. Spontaneous echocardiographic contrast (SEC) was observed in his LA and left ventricle. Mild tricuspid regurgitation and mild pulmonary hypertension were identified with a peak pulmonary systolic pressure of $26 \mathrm{mmHg}$ (estimation based on a peak tricuspid regurgitation jet velocity of $210 \mathrm{~cm} /$ second and an estimated right atrial pressure of $5 \mathrm{mmHg}$ ). His inferior vena cava was not dilated and collapsible with respiration, and there was no thrombus. His ejection fraction was estimated as $45 \%$.

Patients with severe MS and AF are at high risk of developing intracardiac thrombi. Based on the findings by TTE, TEE, and the presence of risk factors (AF), his RA mass was likely to be a thrombus as described earlier. Similar features were found in LA mass and it was thought to be a thrombus as well. However, the characteristic of LA mass adhering to atrial septum mimics atrial myxoma, which is the most common benign cardiac tumor [1]. The shape, mobility, and location of the LA mass made it difficult to rule out atrial myxoma with absolute certainty. Therefore, our patient underwent CMR for additional noninvasive characterization of the biatrial mass. CMR showed a giant right atrial mass measuring $5.3 \times 3.2 \times 3.9 \mathrm{~cm}$ and a large left atrial lesion measuring $5.0 \times 2.4 \times 5.1 \mathrm{~cm}$ adhering to interatrial septum (Fig. 2). Both lesions were intermediate in signal on cine CMR, and not enhanced during early and delayed enhancement CMR. The morphology, location, and avascular characteristics made thrombi the most likely diagnosis. The CMR result was in agreement with TTE and TEE findings.

Based on his history, physical examinations and imaging findings, we recommended thrombectomy and mitral valve replacement. Despite medical advice, our patient declined surgery. We empirically initiated heparin and warfarin administered intravenously to prevent thromboembolism. His international normalized ratio (INR) was targeted at 2.5 to 3.5 . Bisoprolol was prescribed for AF rate control. However, his clinical course was complicated by his non-adherence to warfarin. His INR was subtherapeutic (median=1.8). After 2 years of warfarin (partial compliance), serial TTE showed no regression of LA and RA masses. After 3 years of uneventful follow-up, he experienced an episode of transient ischemic attack in February 2014. Subsequently, in December 2014, he had an episode of non-fatal ischemic stroke manifested by right hemiparesis (motor power reduced to 3/5) most probably because of thromboembolism. We counseled him with regard to the danger of the thromboembolism and re-emphasized the need for treatment compliance. Fortunately, he resumed warfarin and did not demonstrate further thromboembolic events. In March 2016 (5 years after the detection of biatrial masses), a follow-up TTE documented complete regression of the LA mass and a near-complete resolution of the RA mass (Fig. 3). Thus, the most probable diagnosis of cardiac thrombi was affirmed. He remains well on medical treatment.

\section{Discussion}

Many cases of cardiac masses have been reported in the literature, but in this case report we described a rare case of biatrial cardiac mass that represented a challenge for diagnosis and therapy. In our patient, the characteristic of a left 


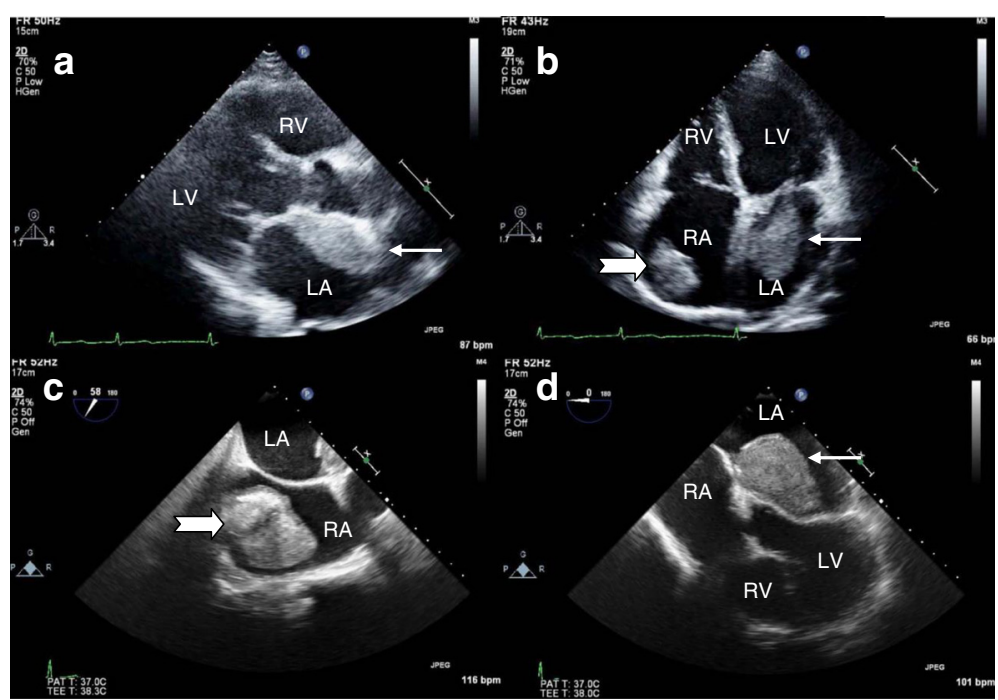

Fig. 1 Images obtained by transthoracic echocardiography and transesophageal echocardiography. a, b Transthoracic echocardiography images. c, d Transesophageal echocardiography images. A large left atrial mass (arrows) adhering to interatrial septum in parasternal long axis (a) and apical four chamber view (b, d) and a giant right atrial mass (notched arrows) was seen in apical four chamber (b) and bicaval view (c). LA left atrium, $L V$ left ventricle, $R A$ right atrium, $R V$ right ventricle

atrial mass adhering to the atrial septum posed a diagnostic challenge in differentiating between thrombus and myxoma, the most commonly reported cardiac masses. The following discussion will review cases of biatrial masses in patients with MS reported between 2008 and 2016 (Table 1). First, a 40-year-old woman with rheumatic MS was found to have biatrial thrombi mimicking myxoma, and she underwent a successful thrombectomy and valve replacement [8]. Second, a 77-year-old man with AF, severe MS and heart failure presented with dyspnea. TEE and TTE revealed biatrial thrombi confirmed by pathological examination following thrombectomy and mitral valve replacement [9]. Third, a 58-year-old woman presented with acute limb ischemia; she was found to have mobile biatrial thrombi, AF, and MS and underwent successful embolectomy, thrombectomy, and mitral valve replacement [10]. The features of biatrial thrombi, MS, and $\mathrm{AF}$ were common to all three patients. In addition, a case of biatrial myxoma with mild MS presented with cerebral ischemia was successfully treated with thrombolytic therapy administered intravenously and surgical resection [11]. However, numerous cases of cardiac mass with or without mitral valve disease with diagnostic difficulties have been reported earlier. Overall, cardiac thrombi were frequently reported $[5,7,10,12,13]$. In some instances a cardiac thrombi mimicked atrial myxoma $[5,7]$. By contrast, atrial myxoma can simulate thrombus in the setting of MS [14]. Of interest, a thrombus could form on top of a myxoma. In one case report of a patient with MS, the left atrial mass showed features of thrombus

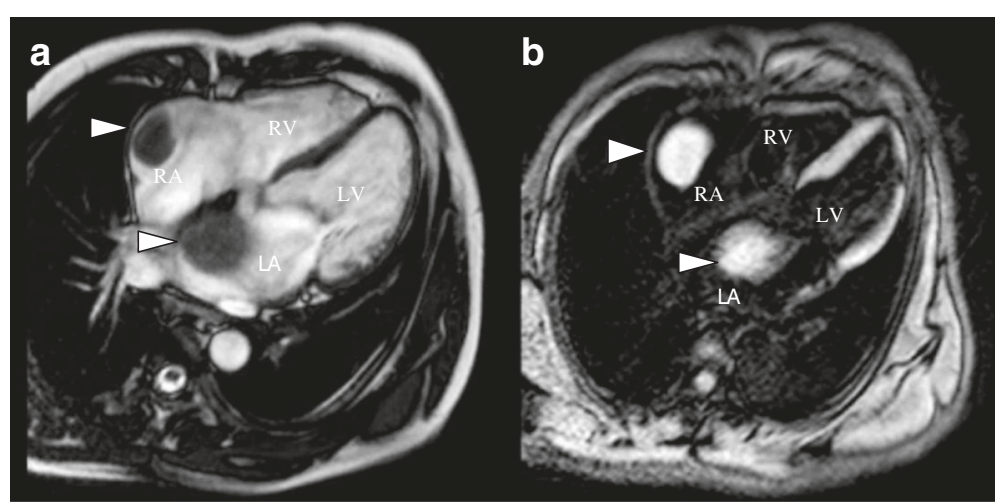

Fig. 2 Cardiac magnetic resonance. Cine four chamber view: "white blood" gradient echo (a) and "black blood" imaging (b) on early gadolinium images showed a low signal floating lesion in the right atrium and a left atrial lesion adhering to the interatrial septum (white triangles). LA left atrium, $L V$ left ventricle, $R A$ right atrium, $R V$ right ventricle 


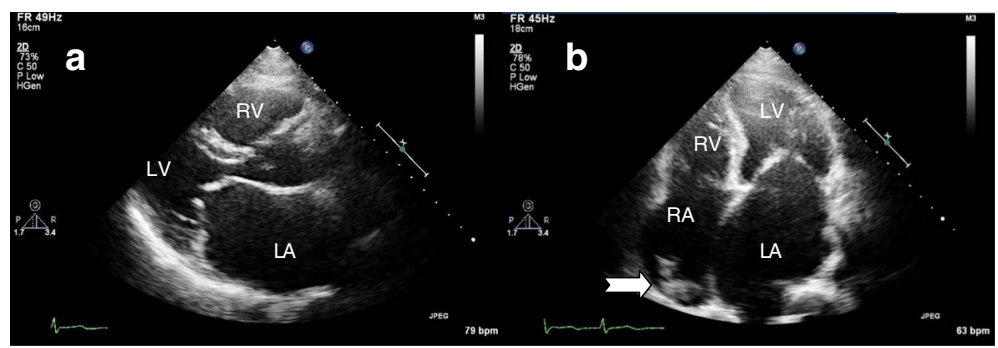

Fig. 3 Outcome after long-term anticoagulation therapy. The left atrial thrombus is completely resolved as viewed from parasternal long axis (a) and apical four chamber view (b) in the left atrium. A residual right atrial thrombus (notched arrow) is still visible in the right atrium (b). LA left atrium, $L V$ left ventricle, $R A$ right atrium, $R V$ right ventricle

characterized by echocardiography and CMR. However, histopathological evaluation of the left atrial mass removed during surgery revealed a massive thrombus formed on top of a very small pre-existing left atrial myxoma [12]. To complicate matters further, atrial thrombus may have a stalk [6] or neovascularization [13] mimicking atrial myxoma, potentially leading to a delay in anticoagulation therapy. In another case report, biatrial intracardiac masses were detected by three-dimensional TEE in an 80-year-old woman with heart failure, mitral valve repair, dual chamber permanent pacemaker implantation, and AF. Although direct pathological specimens were not obtained, the reduction in the size of both masses after intensive anticoagulation treatment raises the strong possibility that both masses were thrombi [15]. This case report highlighted the facts that anticoagulation is a feasible treatment in regressing thrombi when surgery is relatively contraindicated in an older patient with comorbidities.

TEE is superior to TTE in delineating and characterizing cardiac masses [2]. CMR provides high spatial resolution images, improves tissue characterization and is complementary to echocardiography in the assessment of cardiac masses [3]. Common CMR sequences are cine image, T1-weighted and T2-weighted spin echo, contrast first pass perfusion, and standard delay enhancement. However, tumors and chronic organized thrombi cannot be distinguished from one another using the morphology, motility, and enhancement patterns by CMR. A pattern of hyperintensity/isointensity (compared with normal myocardium) with short $\mathrm{T} 1$, and hypointensity with long $\mathrm{T} 1$, was very frequent in thrombi, rare in tumors, and had the highest accuracy for the differentiation of both entities [4]. In addition to conventional imaging studies, the assessment of vascularity either by myocardial perfusion contrast echocardiography [16] or cardiac catheterization may assist in the differentiation of thrombi and other type of cardiac tumors. Nevertheless, in a few instances, the final diagnosis of cardiac masses can only be made by obtaining a pathological specimen after surgical resection of cardiac masses [5-7, 12].

For our patient, the nature of biatrial mass was not specified by histology because he declined surgery. The presence of MS, AF, SEC and dilated LA indicated a diagnosis of thrombi. Our case is unusual because the final diagnosis was affirmed by empirical anticoagulation based on clinical judgment and noninvasive characterization of biatrial mass. A reported case of left atrial thrombus with a stalk showed that a trial of anticoagulation was beneficial in regressing the cardiac mass particularly when the differential diagnosis was difficult and thrombus was a possibility [6]. There were also cases that reported successful regressions of the thrombus with anticoagulation without the need for surgical thrombectomy $[15,17,18]$. Regression of thrombus using warfarin has been studied in patients with MS and left atrial thrombus detected prior to percutaneous transvenous mitral commissurotomy. Among 219 patients following 6 months of warfarin (INR 2 to 3 ) therapy, $24.2 \%$ of patients were found to have complete resolution of thrombus and $75.8 \%$ of patients were found to have partial resolution of thrombus, and a higher INR (at least 2.5) predicted thrombus resolution [19]. For our patient, the regression of his cardiac masses was not observed in the short term after treatment with

Table 1 Biatrial mass with mitral stenosis

\begin{tabular}{lllllll}
\hline Author & Year & Age/Sex & Presentation & Atrial fibrillation & Diagnosis & Treatment \\
\hline Ibrahim et al. [11] & 2008 & $51 /$ male & Right hemiparesis & - & Myxoma & Alteplase administered intravenously + tumor excision \\
Tasdemir et al. [10] & 2008 & $58 /$ female & Left foot pain & + & Thrombi & Thrombectomy + MV surgery \\
Tsubokawa et al. [9] & 2010 & $77 /$ male & Dyspnea & + & Thrombi & Thrombectomy + MV surgery \\
Khanna et al. [8] & 2015 & $40 /$ female & Dyspnea & + & Thrombi & Thrombectomy + MV surgery
\end{tabular}


warfarin, casting doubt on the true identity of the biatrial mass. We suspect the main contributing factor towards the lack of thrombi regression was inadequate anticoagulation owing to his lack of compliance. For this reason, compliance to anticoagulation therapy was pivotal in thrombi regression, and it took 5 years to visualize the resolution of biatrial thrombi. Although a thorough attempt had been made, we could not convince our patient to agree to surgery. We believe that early mitral valve surgery and thrombectomy is beneficial in preventing thromboembolism.

\section{Conclusions}

Noninvasive characterization of cardiac mass is essential in clarifying the diagnosis and directing treatment strategy. Anticoagulation is a feasible treatment when the clinical assessment, risk factors, and imaging findings indicate a diagnosis of thrombi. After prolonged anticoagulation therapy, complete resolution of biatrial thrombi was achievable in our case.

\section{Abbreviations}

$A F$, atrial fibrillation; CMR, cardiac magnetic resonance; INR, international normalized ratio; LA, left atrium; MS, mitral stenosis; RA, right atrium; SEC, spontaneous echocardiographic contrast; TEE, transesophageal echocardiography; TTE, transthoracic echocardiography

\section{Acknowledgements}

Not applicable.

\section{Funding}

Self-funded.

\section{Availability of data and supporting materials}

Not applicable.

\section{Authors' contributions}

$H T L$ is the first author who treated the patient, organized the investigations, and obtained patient consent. HTL and RN wrote the first draft of manuscript. CNC, THG, and NO were involved in acquisition and interpretation of images. BCL, JYK, and GR treated the patient and provided comments on the manuscript. All authors read and approved the final manuscript.

\section{Competing interests}

The authors declare that they have no competing interests.

\section{Consent for publication}

Written informed consent was obtained from the patient for publication of this case report and accompanying images. A copy of the written consent is available for review by the Editor-in-Chief of this journal.

\section{Author details \\ ${ }^{1}$ Clinical School Johor Bahru, Jeffrey Cheah School of Medicine and Health Sciences, Monash University Malaysia, 8 Jalan Masjid Abu Bakar, 80100 Johor Bahru, Johor, Malaysia. ${ }^{2}$ Department of Radiology, Sultanah Aminah Hospital, Jalan Abu Bakar, 80100 Johor Bahru, Johor, Malaysia. ${ }^{3}$ Department of Cardiology, Sultanah Aminah Hospital, Jalan Abu Bakar, 80100 Johor Bahru, Johor, Malaysia. ${ }^{4}$ Department of Cardiology, Penang General Hospital, Jalan Residensi, 10990 Georgetown, Pulau Pinang, Malaysia.}

Received: 27 June 2016 Accepted: 28 July 2016

Published online: 10 August 2016

\section{References}

1. Bruce CJ. Cardiac tumours: diagnosis and management. Heart. 2011:97:151-60.

2. Manning WJ, Weintraub RM, Waksmonski CA, et al. Accuracy of transesophageal echocardiography for identifying left atrial thrombi: a prospective, intraoperative study. Ann Intern Med. 1995;123(11):817-22.

3. Altbach MI, Squire SW, Kudithipudi V, et al. Cardiac MRI is complementary to echocardiography in the assessment of cardiac masses. Echocardiography. 2007;24:286-300.

4. Pazos-López P, Pozo E, Siqueira ME, et al. Value of CMR for the differential diagnosis of cardiac masses. JACC Cardiovas Imaging. 2014;7(9):896-905.

5. Kodali S, Yamrozik J, Biederman RWW. Left atrial thrombus masquerading as a myxoma in a patient with mitral stenosis: case report. Echocardiography. 2010;27:98-101.

6. Jang $\mathrm{KH}$, Shin $\mathrm{DH}$, Lee $\mathrm{CK}$, et al. Left atrial mass with stalk: thrombus or myxoma? J Cardiovasc Ultrasound. 2010;18(4):154-6.

7. Dhawan S, Tak T. Left atrial mass. Thrombus mimicking myxoma. Echocardiography. 2004;21(7):621-3.

8. Khanna SN, Paul M, Sharma V, et al. A case of biatrial mass mimicking myxoma with rheumatic mitral stenosis with regurgitation of moderate severity. J Ind Coll Cardio. 2015;5(4):338-40.

9. Tsubokawa K, Muramoto A, Tarui T, et al. Unusual biatrial thrombus in mitral stenosis. J Echocardiogr. 2010;8(4):135-6.

10. Tasdemir K, Sarlib B, Kayab MG, et al. Mobile biatrial thrombus in a patient with mitral stenosis under heparin infusion. Inter CardioVas Thor Surg. 2008; 7:667-9.

11. Ibrahim M, Iliescu C, Safi HJ, et al. Biatrial myxoma and cerebral ischemia successfully treated with intravenous thrombolytic therapy and surgical resection. Tex Heart Inst J. 2008;35(2):193-5.

12. Deluigi CC, Meinhardt G, Ursulescu A, et al. Noninvasive characterization of left atrial mass. Circulation. 2006;113:19-20.

13. Acet $H$, Duygu $H$, Ertas F, et al. A case of mobile giant left atrial thrombus which vascularized with coronary arteries in severe mitral valve stenosis. Cardiovasc Revasc Med. 2010;11:137-8.

14. Mahdhaoui A, Bouraoui $H$, Amine MM, et al. The transesophageal echocardiographic diagnosis of left atrial myxoma simulating a left atrial thrombus in the setting of mitral stenosis. Echocardiography. 2004;21(4):333-6.

15. Imamura $\mathrm{K}$, Takeuchi $\mathrm{M}$, Haruki N, et al. Simultaneous visualization of two intracardiac masses in both atria on 3D transesophageal echocardiography. Circulation J. 2011;75(4):986-8.

16. Kirkpatrick JN, Wong T, Bednarz JE, et al. Differential diagnosis of cardiac masses using contrast echocardiographic perfusion imaging. J Am Coll Cardiol. 2004:43:1412-9.

17. Khan H, Chaubey S, Hill A, et al. Large left atrial thrombus after mitral valve replacement: how to treat? Asian Cardiovasc Thorac Ann. 2012;20:457-9.

18. Al-Bezem R, Kwan T, Clark LT. Regression of large atrial thrombi and coronary neovascularizations with conventional anticoagulation in mitral stenosis, a case report. Angiology. 1999;50(10):859-63.

19. Silaruks S, Thinkhamrop B, Kiatchoosakun S, et al. Resolution of left atrial thrombus after 6 months of anticoagulation in candidates for percutaneous transvenous mitral commissurotomy. Ann Int Med. 2004;140:101-5.
Submit your next manuscript to BioMed Central and we will help you at every step:

- We accept pre-submission inquiries

- Our selector tool helps you to find the most relevant journal

- We provide round the clock customer support

- Convenient online submission

- Thorough peer review

- Inclusion in PubMed and all major indexing services

- Maximum visibility for your research

Submit your manuscript at www.biomedcentral.com/submit
CioMed Central 